\title{
Phosphore et bisphosphonates : ou quand on oublie les leçons du passé !
}

\section{Phosphorus and bisphosphonates: or the forgotten lessons of the past!}

\author{
JEAN-PIERRE CARREL*, SEEMAN ABI NAJM*, STELLA LYSITSA*, \\ PHILIPPE LESCLOUS**, TOMMASO LOMBARDI*, JACKY SAMSON*
}

\begin{abstract}
RÉSUMÉ
La nécrose osseuse des maxillaires, qui se développe chez des patients ayant été traités par des bisphosphonates, constitue une complication tardive rapportée pour la première fois en 2003. L'imputabilité des bisphosphonates a été difficile à établir pour deux raisons principales : l'étiopathogénie des lésions n'est pas totalement élucidée et l'industrie pharmaceutique a tardé à admettre l'origine de cette complication, l'attribuant à la chimiothérapie anticancéreuse et/ou à des actes de chirurgie buccale. Une affection similaire a été décrite il y a presque deux siècles chez des travailleurs de l'industrie des allumettes exposés au phosphore blanc. L'étiopathogénie de ces ostéonécroses appelées «phossy jaw » n'a jamais été totalement élucidée, mais l'imputabilité du phosphore ne faisait aucun doute, malgré le délai parfois important entre l'exposition au phosphore et l'apparition des lésions. La prise en considération de ces données aurait sans doute incité à plus de prudence avant la mise sur le marché des bisphosphonates et à mieux préciser la posologie et les indications. (Med Buccale Chir Buccale 2006; 12: 7-14)
\end{abstract}

mots clés : bisphosphonates, ostéonécrose, phosphates, phosphore, phossy jaw

médecine buccale chirurgie buccale

VOL. $12, \mathrm{~N}^{\circ} 1$ 2006

page 7

\section{SUMMARY}

Osteonecrosis of the jaws developing as a late complication in patients treated with bisphosphonates was first described in 2003. The relationship between bisphosphonates and osteonecrosis has been difficult to establish for two main reasons: firstly because the aetiopathology of the lesion still remains unclear and secondly because of the refusal of the pharmaceutical industry to recognise this class of drugs as the aetiopathological factor. Pharmaceutical companies, on the contrary, suggested cancer chemotherapy or oral surgery procedures as primary causes of osteonecrosis of the jaw.

Interestingly, a similar disease was described approximately 2 centuries ago among match industry workers who were exposed to phosphorus. This clinical entity was named "phossy jaw". Although the pathogenesis of this form of osteonecrosis was never completely clarified, it was without any doubt attributed to chronic exposure to white phosphorus. Upon careful evaluation of the scientific data, more attention should have been focused on the posology and indications of bisphosphonates and their side effects, before their release on the market. (Med Buccale Chir Buccale 2006; 12: 7-14)

key words : bisphosphonates, osteonecrosis, phosphate, phosphorus, phossy jaw

\footnotetext{
* Division de Stomatologie, Chirurgie Orale et Radiologie Dento-Maxillo-Faciale, Faculté de Médecine, Genève, Suisse

** Faculté de Chirurgie Dentaire, Paris 5, France

Demande de tirés à part :

Jean-Pierre Carrel Division de Stomatologie Chirurgie Orale et Radiologie Dento-Maxillo-Faciale Faculté de Médecine 19, rue Barthélémy-Menn 1205 Genève Suisse jean-pierre.carrel@medecine.unige.ch
} 
médecine

buccale

chirurgie

buccale

VOL. $12, \mathrm{~N}^{\circ} 1$ 2006

page 8
L'ostéonécrose des maxillaires représente un effet indésirable des bisphosphonates décrit pour la première fois en 2003, par Marx et al. [1]. Son étiopathogénie n'est pas totalement élucidée, mais il semble qu'elle soit la conséquence d'un déficit de la vascularisation, secondaire à une minéralisation osseuse excessive [1,2]. Les lésions intéressent exclusivement les maxillaires et leur point de départ est toujours intra-buccal. La cavité buccale représente une région anatomique et physiologique unique dans l'organisme humain : la présence de dents reliées à l'os par un ligament, la particularité de l'attache épithéliale sur le collet des dents, la fréquence des microtraumatismes lésant la muqueuse et la présence permanente de micro-organismes constituent autant d'éléments qui, lors d'une inflammation ou d'une lésion gingivale, favorisent le contact entre l'os et le milieu septique buccal [2,3]. Lorsque l'os est hyperminéralisé, donc moins bien vascularisé, sa capacité de défense est réduite et une ostéite peut plus facilement se développer. $\mathrm{Ce}$ phénomène est bien connu dans certaines maladies ostéocondensantes comme l'ostéopétrose ou la maladie de Paget [2,4]. L'ostéonécrose due aux bisphosphonates en est une nouvelle illustration. Seul un faible nombre de patients traités par bisphosphonates semblent développer une ostéonécrose, ce qui suggère que des facteurs individuels, peut-être immunitaires, pourraient jouer un rôle important. Des fractures pathologiques des os longs avec retard de consolidation, principalement des fémurs, ont été également rapportées: elles seraient dues à la modification de la compliance de l'os hyperminéralisé [5].

Des lésions osseuses similaires, survenant chez des travailleurs de l'industrie des allumettes exposés au phosphore blanc, ont été décrites il y a presque deux siècles $[3,5,6]$. Cette ostéonécrose se présentait sous une forme identique à celle induite par les bisphosphonates. II s'agissait d'expositions osseuses toujours intra-buccales initialement, douloureuses ou non, évoluant en absence de traitement vers l'extension progressive dans les régions péri-buccales et cervicales, aboutissant parfois à des délabrements faciaux importants. Le taux de mortalité de cette affection variait de $10 \%$ à $40 \%$ avant l'ère des anti- biotiques [5,6]; le décès était habituellement secondaire à une infection. Plusieurs cas de suicide ont été rapportés chez des malades qui ne pouvaient plus tolérer leur propre image $[5,6]$. Dans d'autres cas, les lésions évoluaient très lentement et parfois même se stabilisaient. Le seul traitement efficace consistait en une exérèse chirurgicale de l'os nécrosé permettant quelquefois, si elle était réalisée de manière précoce, une cicatrisation des lésions [5,6]. L'industrie des allumettes au phosphore employait, à la fin des années 1800 , plusieurs dizaines de milliers d'individus et seul un petit nombre (2-3\%) ont développé ces lésions [3,5]. L'étiopathogénie de la maladie n'a jamais été formellement élucidée. Une hygiène bucco-dentaire insuffisante et un état dentaire négligé semblaient favoriser l'apparition des lésions [3,5,6]. Les manifestations apparaissaient plusieurs mois, voire plusieurs années, après le début de l'intoxication au phosphore, parfois même plusieurs années après l'arrêt de l'exposition à cette substance $[3,5]$. Ces constatations contredisaient les hypothèses pathogéniques initiales qui évoquaient une action locale et directe du phosphore. On avait également constaté que des fractures pathologiques des os longs pouvaient survenir plusieurs années ou même quelques décennies après la fin de l'exposition: ceci confirmait que le remaniement osseux touchait l'ensemble du squelette [5].

\section{RAPPEL HISTORIQUE}

Il semble que l'alchimiste arabe Alchid Bechil (XII ${ }^{e}$ siècle) connaissait déjà le phosphore. Officiellement, il a été découvert en 1669 dans l'urine humaine par Henning Brand, un alchimiste allemand également à la recherche de la pierre philosophale. II a isolé cet élément en traitant une grande quantité d'urine par évaporations et distillations successives. Enfin par chauffage prolongé, il a constaté la présence d'une matière solide déposée sur la paroi de ses cornues. Ce dépôt présentait la caractéristique d'émettre une lumière blanchâtre dans le noir en présence d'air (d'où le nom de phosphore: du grec phos, lumière et phoros, celui qui porte). Vers 1690, l'anglais Robert Boyle a amélioré le procédé: I'adjonction 
de silicate donne des scories, à partir desquelles il était plus facile d'obtenir le phosphore. II est maintenant extrait de roches ayant un fort contenu en phosphates. Ces roches existent partout dans le monde et le phosphore représente quantitativement le onzième élément parmi les constituants de la croûte terrestre. II existe sous 3 formes allotropiques: le phosphore blanc ou jaune, très toxique qui s'enflamme spontanément à $34^{\circ} \mathrm{C}$ en présence d'oxygène, le phosphore rouge obtenu à partir du précédent par chauffage prolongé et le phosphore noir ou violet par chauffage très intense sous pression élevée; les deux dernières formes ne sont pas toxiques [7].

La capacité d'inflammation du phosphore blanc est à l'origine de l'industrie des allumettes, de la pyrotechnie, et plus tard il fut utilisé dans l'armement. En 1831, le médecin français Charles Sauria invente l'allumette à friction. L'année suivante, la fabrication industrielle des allumettes commence à Vienne en Autriche sous l'impulsion de l'allemand Jakob Friedrich Kammerer. En 1839, le premier cas d'ostéonécrose des maxillaires est rapporté chez un sujet travaillant dans l'usine d'allumettes viennoise par Friedrich Wilhelm Lorinser $[3,5,6]$. En quelques années, plusieurs manufactures fabricant des allumettes seront construites en Europe, parallèlement le nombre de cas de nécrose des maxillaires chez les travailleurs du phosphore ne cesse de croître. Le terme «Kieferphosphornekrose» des auteurs allemands devient pour les auteurs anglophones «phossy jaw », terme qui sera repris en médecine. Cette maladie fut rapidement reconnue comme une maladie professionnelle: l'imputabilité du phosphore dans le développement des lésions ne faisait aucun doute puisque seuls les travailleurs en contact ou ayant été en contact avec le phosphore blanc développaient de telles atteintes. En 1872, la Finlande fut le premier pays à interdire l'utilisation du phosphore blanc dans l'industrie, imitée par d'autres pays, comme la Suisse en 1898. La convention de Berne interdisant l'utilisation du phosphore pour la fabrication des allumettes a été signée en 1906 par la majorité des pays européens. Pendant la période 1939-1945, l'augmentation de l'utilisation du phosphore blanc dans l'industrie militaire, plus particulièrement en
Allemagne, a entraîné l'apparition d'un grand nombre de nouveaux cas d'ostéonécrose des maxillaires [3]. L'introduction de normes de sécurité au travail a eu pour conséquence une diminution importante des cas. Depuis les années 60, les observations rapportées dans la littérature sont exceptionnelles [3].

\section{Phosphates}

Le phosphore est un élément très réactif qui intervient dans de nombreuses réactions chimiques. Les produits dérivés de ces réactions sont bien connus tant du point de vue de la chimie que de celui de leurs propriétés. Les plus importants sont sans doute les dérivés de l'acide phosphorique ou phosphates: deux molécules d'acide phosphorique se lient entre elles par réaction anhydre d'acide réalisant une liaison $\mathrm{P}-\mathrm{O}-\mathrm{P}$ avec formation d'une molécule d' $\mathrm{H}_{2} \mathrm{O}$. La liaison aboutit à la formation d'un diphosphate appelé pyrophosphate (Tab. 1). Ces produits sont indispensables à la vie végétale et animale. Les spécialistes de l'agriculture savent depuis longtemps que la production des végétaux est plus importante dans les terres riches en phosphates: ils sont donc utilisés depuis longtemps comme fertilisants. Les propriétés anticorrosives des polyphosphates sont aussi connues depuis plusieurs décennies, d'où leur utilisation pour l'entretien des circuits hydrauliques. Dans les années 30, lors d'une erreur de manipulation, de faibles quantités de polyphosphates ont été déversées de manière

Tableau 1 : Synthèse des polyphosphates. Polyphosphates synthesis<smiles>O=P([O-])([O-])[O-]</smiles>

phosphate inorganique

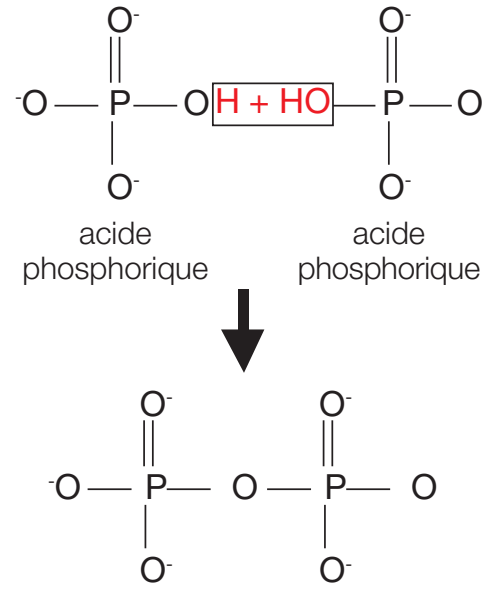

pyrophosphate médecine

buccale

chirurgie

buccale

VOL. $12, N^{\circ} 1$ 2006

page 9 
médecine

buccale

chirurgie

buccale

VOL. $12, \mathrm{~N}^{\circ} 1$ 2006

page 10 continue dans un système d'arrosage agricole. Les conduites ayant reçu des polyphosphates ne présentaient aucun dépôt de tartre après une utilisation prolongée. Cette propriété a été confirmée par le monde scientifique et appliquée pour la réalisation des appareils adoucissant l'eau potable. En médecine, l'importance des phosphates est bien connue: ils entrent dans la composition des acides nucléiques, des os et des dents et ils jouent un rôle primordial dans les réactions biochimiques de transfert d'énergie (système adénosine mono, di et triphosphate). La médecine s'est aussi intéressée à la propriété inhibitrice de la calcification par les dérivés phosphatés, particulièrement pour prévenir les calcifications hétérotopiques des tissus mous. Les propriétés anticalcifiantes de l'urine étaient connues, mais ce n'est qu'en 1960 que le suisse Herbert Fleisch découvrit que cet effet était dû aux pyrophosphates [8]. Cependant, I'utilisation de cette molécule pour prévenir la formation des plaques d'athérome, la calcification des valves cardiaques et la formation des calculs urinaires a été un échec. Fleisch a remarqué que les pyrophosphates étaient rapidement hydrolysés par une enzyme, la pyrophosphatase, largement présente dans les tissus de l'organisme.

\section{Phosphonates}

Ce sont des dérivés de l'acide phosphonique (Tab. 2). Ils ont à l'inverse des phosphates une tendance à former des liaisons $\mathrm{P}-\mathrm{C}-\mathrm{P}$, ce qui les rend résistants à l'hydrolyse [2,8,9]. Les bisphosphonates sont aussi connus depuis longtemps; ils ont été synthétisés pour la première fois par le chimiste russe Nikolai Alexandrovitch Menschutkin en 1865. La synthèse de l'étidronate, qui toujours

Tableau 2 : Formule de l'acide phosphonique et du phosphonate.

Phosphoric acid and phosphate formula.<smiles>[R]P(=O)(O)O</smiles><smiles>[R]OP([R])(=O)O[R]</smiles>

utilisé en médecine, a été réalisée par Adolf von Baeyer et August Wilhelm von Hoffmann en 1897 [8]. Les bisphosphonates sont des analogues structuraux du pyrophosphate inorganique (Tab. 3). L'équilibre chimique nécessite la liaison de 2 radicaux (R1 et R2) sur l'atome de carbone, ce qui offre un nombre presque illimité de combinaisons différentes. Certains radicaux confèrent des propriétés particulières aux bisphosphonates. On distingue deux grandes classes: les composés simples comportant $\mathrm{CH}, \mathrm{CH} 3$ ou $\mathrm{H}$ en position $\mathrm{R} 2$ et ceux qui possèdent un atome d'azote dans leur radical $\mathrm{R} 2$ ou aminobisphosphonates, encore appelés dérivés nitrogéniques (Tab. 4), qui ont un mode d'action différent et qui sont plus puissants $[2,8,9,10]$.

Les bisphosphonates ont été utilisés en médecine sans résultats probants pour la première fois par Herbert Fleisch en 1968, pour inhiber les calcifications hétérotopiques [8]. On pensait que la résistance à l'hydrolyse leur permettrait d'avoir une importante action d'inhibition de la calcification des tissus mous. Ces molécules ont montré une très forte affinité pour l'hydroxyapatite. Ils ne sont pour la plupart pas métabolisés, et seule la partie qui n'a pas adhéré à l'os est rapidement éliminée sous forme inchangée par le rein. Les scientifiques ont remarqué que les bisphosphonates liés à l'os modifiaient le métabolisme osseux, principalement en inhibant la résorption osseuse. Cette propriété offrait des perspectives intéressantes pour le traitement de plusieurs maladies osseuses. L'industrie pharmaceutique a rapidement perçu l'intérêt de ce marché et plusieurs molécules nouvelles, toujours plus puissantes, sont apparues sur le marché. La puissance des molécules a été établie selon le

Tableau 3 : Analogie de la structure des pyrophosphates et de celle des bisphosphonates.

Structural analogy between pyrophosphates and bisphosphonates.<smiles>O=P(O)(O)OP(=O)(O)O</smiles><smiles>[R]C([R])(P(=O)(O)O)P(=O)(O)O</smiles> 
Tableau 4 : Bisphosphonates commercialisés : structure moléculaire et puissance (d'après Bartl R [11]) Commercially available bisphosphnates: molecular structure and potency

\begin{tabular}{|c|c|c|c|c|}
\hline $\mathrm{DCl}$ & Nom commercial & R1 & $\mathbf{R} 2$ & Puissance \\
\hline Etidronate & Didronel® & $\mathrm{OH}$ & $\mathrm{CH}_{3}$ & 1 \\
\hline Clodronate & Clastoban ${ }^{\circledR}$ & $\mathrm{Cl}$ & $\mathrm{Cl}$ & 10 \\
\hline Tiludronate & Skelid® & $\mathrm{H}$ & S- CL & 10 \\
\hline Pamidronate & Arédia & $\mathrm{OH}$ & $\mathrm{CH}_{2}-\mathrm{CH}_{2}-\mathrm{NH}_{2}$ & 100 \\
\hline Alendronate & Fosamax® & $\mathrm{OH}$ & $\mathrm{CH}_{2}-\mathrm{CH}_{2}-\mathrm{CH}_{2}-\mathrm{NH}_{2}$ & $1 ' 000$ \\
\hline Risédronate & Actonel@ & $\mathrm{OH}$ & $\mathrm{CH}-\mathrm{N}$ & 5,000 \\
\hline Ibandronate & Bondronat ${ }^{\circledR}$ & $\mathrm{OH}$ & $\mathrm{CH}_{2}-\mathrm{CH}_{2}-\mathrm{N} . \mathrm{C}_{5} \mathrm{H}_{11}$ & $10^{\prime} 000$ \\
\hline Zolédronate & Zométa® & $\mathrm{OH}$ & $\mathrm{CH}_{2}-\mathrm{N} \mathrm{N}$ & $20 \prime 000$ \\
\hline
\end{tabular}

modèle de Schenk : le traitement par bisphosphonates de jeunes rats induit, pendant la croissance, une ostéopétrose identique à la forme congénitale. La dose minimale de chaque composé nécessaire pour induire une ostéopétrose est comparée à celle de l'étidronate qui a été prise comme référence. Par exemple, le zolédronate est 10000 fois plus puissant que l'étidronate $[8,10]$ ou 20000 fois plus puissant selon d'autres auteurs [11].

\section{Mécanismes d'action des bisphosphonates}

Les bisphosphonates adhèrent à l'hydroxyapatite et, lors du processus de remodelage osseux, ils sont absorbés par les ostéoclastes dont ils inhibent l'action [9,12]. Ils ont aussi des propriétés antiinflammatoires [13], un effet antitumoral propre [14] et, pour les aminobisphosphonates, une activité anti-angiogénique [15]. Les mécanismes inhibant la résorption osseuse ne sont pas totalement élucidés et ils semblent être différents selon la molécule. Certains bisphosphonates, comme le tiludronate, ont une action sur la pompe à proton ATPase dépendante et empêcheraient la constitution d'un milieu acide nécessaire pour la résorption osseuse dans la zone sous-ostéoclastique. D'autres, comme les aminobisphosphonates, ont une action directe sur l'ostéoclaste, induisant son apoptose par inhibition du cycle du mévalonate. Le cycle du mévalonate aboutit à la géranylgéranylation des protéines, principalement des petites GTPases agissant comme messager intracellu- laire: leur présence constitue un signal antiapoptotique pour diverses cellules dont celles dérivées des macrophages.

Les bisphosphonates semblent aussi activer les ostéoblastes, ce qui provoquerait une augmentation de la minéralisation osseuse. La controverse persiste et certains auteurs [16] doutent que l'activation des ostéoblastes aboutisse réellement à la formation d'os normal. Les examens histomorphométriques de l'os traité par bisphosphonates montrent une prolifération des ostéoblastes probablement dose-dépendante avec, dans certains cas, une formation de substance ostéoïde normale. L'examen macroscopique des pièces d'exérèse d'os nécrosé montre un os anormal, hyperminéralisé, prenant parfois un aspect crayeux, avec diminution ou disparition de l'os spongieux et de la vascularisation. Cette modification de la structure osseuse tend à démontrer que la résorption osseuse est diminuée et que la formation osseuse est augmentée. Le remodelage osseux physiologique dépend classiquement du couplage entre l'action des ostéoclastes et celle des ostéoblastes, aboutissant à la néoformation d'une quantité d'os égale à celle qui a été résorbée et ayant une architecture semblable. L'augmentation de la minéralisation indique que ce couplage est probablement perturbé par les bisphosphonates.

La prévention de l'ossification hétérotopique et de la calcification des tissus mous dépend d'un processus différent, probablement physico-chimique [8,17]. Cette propriété est observée lorsque médecine

buccale

chirurgie

buccale

VOL. $12, \mathrm{~N}^{\circ} 1$ 2006

page 11 
médecine

buccale chirurgie buccale

VOL. $12, \mathrm{~N}^{\circ} 1$ 2006

page 12 la concentration en bisphosphonates est constante. On observe la présence d'une matrice cellulaire d'apparence normale, mais celle-ci ne se calcifie pas. Gollomb et al., en 1986, ont montré que des bioprothèses aortiques implantées dans le derme de rats ne se calcifiaient pas en présence de bisphosphonates alors que, dans le groupe contrôle, on observait une calcification des prothèses [18]. Plus tôt, Hollander et. al avaient montré en 1978 que chez des animaux ayant des lésions d'artériosclérose, la média des artères, épaissie par la maladie, retrouvait une épaisseur normale après un traitement par bisphosphonates à doses élevées et concentration constante [19] .

\section{Indications}

Les principales indications des bisphosphonates sont le traitement de l'hypercalcémie maligne et des métastases osseuses observées dans divers cancers (sein, prostate...) [2,8]. Ils ont grandement amélioré la qualité de vie des malades atteints d'un myélome multiple ou de métastases osseuses en diminuant les douleurs osseuses, l'impotence fonctionnelle et les complications osseuses (fractures spontanées, tassements vertébraux...). Comme leurs effets indésirables immédiats sont presque toujours mineurs et transitoires, on a rapidement multiplié leurs indications. Ils sont actuellement prescrits dans la maladie osseuse de Paget, l'ostéoporose postménopausique, l'ostéoporose cortisonique, la nécrose aseptique de la hanche, l'ostéogenèse imparfaite, le syndrome SAPHO... [2,12]. L'étidronate est prescrit pour la prévention des calcifications hétérotopiques après un traumatisme médullaire ou dans la période suivant la mise en place d'une prothèse totale de la hanche [17].

\section{Effets indésirables}

Ils sont bien connus et considérés comme banaux et transitoires. Le symptôme le plus grave est une insuffisance rénale aiguë mais elle peut être prévenue par une administration intraveineuse lente $[12,20]$. Avec la majorité des bisphosphonates, on observe un syndrome pseudo-grippal avec élévation temporaire de la température, des frissons, une sensation de fatigue, des arthralgies et des myalgies. Ces symptômes régressent habituelle- ment dans les 48 heures suivant l'administration. Des manifestations digestives sont régulièrement rapportées: nausées, vomissements, diarrhées, douleurs abdominales, oesophagite, ulcérations buccales. Des ulcères d'estomac et une sensation de sécheresse buccale sont parfois observés. La formule sanguine est souvent perturbée: anémies, thrombocytopénies, leucopénies et parfois pancytopénies. Les effets indésirables neurologiques se traduisent par des céphalées, des vertiges, des sensations d'hypoesthésie ou à l'inverse d'hyperesthésie, et de dysgueusies. Le système immunitaire peut être aussi concerné: des rares cas d'hypersensibilité immédiate et d'érythème polymorphe ont été notés. Une altération de la réponse immunitaire a été démontrée lors de la prescription d'étidronate sous la forme de réactivation fréquente d'herpès secondaires et de zona. De rares cas de manifestations oculaires à type d'uvéite et de conjonctivite peuvent survenir. Les anomalies métaboliques du type hypocalcémie sont le plus souvent asymptomatiques, parfois elles peuvent induire des crampes musculaires ou des épisodes de tétanie.

En 2003, les premiers cas d'ostéonécrose des maxillaires en relation avec la prise de bisphosphonates ont été décrits. Cet effet indésirable tardif, qui ne survient qu'après plusieurs mois ou années de traitement, semble concerner seulement une partie des malades traités, mais le recul est sans aucun doute insuffisant pour évaluer la fréquence de cette complication.

\section{DISCUSSION}

L'étiologie du «phossy jaw » n'a jamais été totalement élucidée, mais le phosphore a rapidement été reconnu comme l'élément déclenchant de l'ostéonécrose des maxillaires. Dans le contexte de l'époque, les autorités médicales ont réagi de manière appropriée et avec une rapidité remarquable, en reconnaissant cette affection comme une maladie professionnelle et en sensibilisant les autorités politiques pour les amener à prendre des mesures. L'interdiction de l'utilisation du phosphore blanc fut décrétée dans toute l'Europe. Dans le cas des bisphosphonates, la réaction des 
autorités médicales, y compris des organismes de pharmacovigilance, a été tardive et probablement bien timorée [21,22,23]. L'ostéonécrose des maxillaires chez les patients traités par bisphosphonates est une complication peut-être rare, mais potentiellement dramatique, qui semble avoir été sous-estimée par les autorités médicales. Cette attitude frileuse est compréhensible: ceux qui ont pris les décisions n'ont probablement jamais examiné un cas d'ostéonécrose, ignorent les phossy-jaws et peinent à reconnaître que leur (pharmaco-) vigilance a été de nouveau prise en défaut. L'imputabilité des bisphosphonates a été initialement éludée, car les premiers cas sont apparus chez des patients qui présentaient des métastases osseuses; on a naturellement incriminé la chimiothérapie anticancéreuse ou la radiothérapie comme facteurs étiopathogéniques principaux [24,25]. Cette hypothèse convenait parfaitement aux firmes pharmaceutiques, mais l'apparition de lésions similaires chez des malades traités pour une ostéoporose, et par ailleurs en bonne santé, a permis de réfuter cette hypothèse étiopathogénique. La localisation exclusivement maxillaire des lésions a permis à quelques médecins, attachés à la firme pharmaceutique commercialisant les principaux bisphosphonates incriminés, de considérer les gestes bucco-dentaires comme responsables des lésions et ont proposé de manière empirique, sans consulter

\section{RÉFÉRENCES}

1 - Marx RE, Sawatari Y, Fortin M, Broumand V. Bisphosphonate - induced exposed bone (osteonecrosis/osteopetrosis) of the jaws: risk factors, recognition, prevention, and treatment. J Oral Maxillofac Surg 2005 ; 63: 1567-75.

2 - ABi Najm S, Lysitsa S, CarRel JP, Lesclous P, LOMBARDI T, SAMSON J. Ostéonécrose des maxillaires chez des patients traités par bisphosphonates. Presse Med 2005 ; 34: 1073-7.

3 - JAKHI SA, PAREKH BK, GUPTA S. Phosphorus necrosis of the maxilla. J Oral Med 1983 ; 38: 174-6.

4 - Hellstein JW, Marek CL. Bisphosphonate osteochemonecrosis (bis-phossy jaw): is this phossy jaw of the 21st century? J Oral Maxillofac Surg 2005 ; 63: 682-9.

5 - HUGHES JPW, BARON R, BUCKLAND DH, COOKE MA, CRAIG JD, DufField DP, Grosart AW, Parkes PWJ, Porter A. les spécialistes du domaine, d'éviter la réalisation d'actes bucco-dentaires chez ces patients [25]. Cette hypothèse a aussi été rapidement écartée car plusieurs cas de nécroses osseuses spontanées en région édentée ont été rapportés.

La concurrence entre les firmes pharmaceutiques est telle qu'il faut absolument, pour des impératifs financiers, accélérer la mise sur le marché d'un médicament quitte à négliger l'étude d'effets indésirables suspectés ou suspectables; l'exemple des inhibiteurs de la COX 2 est caractéristique de cet état d'esprit [26]. L'apparition d'effets indésirables tardifs nécessite une durée d'observation prolongée lors d'étude chez l'animal, mais il est bien difficile de déterminer la durée idéale des tests. Des études complémentaires doivent rapidement être réalisées pour déterminer l'étiopathogénie de cette ostéonécrose et la posologie optimale des différents bisphosphonates car ces molécules sont peu métabolisées et certaines persistent sous forme active dans l'os plus de 10 ans après une dose unique [20]. L'exemple des «phossy jaws» montre qu'une analyse exhaustive de la littérature conserve une réelle importance mais, en médecine, face à l'évolution exponentielle des connaissances, on a trop tendance à considérer qu'au-delà de 10 ans la bibliographie n'a plus guère d'intérêt. Cette suffisance ne peut conduire qu'à l'ignorance et à son cortège d'erreurs.

Phosphorus necrosis of the jaw : a present-day study. $\mathrm{Br} J$ Ind Med 1962 ;19: 83-99.

6 - KraBBE KH. Zur Frühgeschichte des Kampfes gegen die Phosphornekrose in Deutschland. Z Gesamte Hyg 1976 ; 22: 447-54.

7 - http://www.periodictableonline.org

8 - FLEISCH H. Bisphosphonates: mechanisms of action. Endocr Rev 1998 ; 19: 80-100

9 - ROGERS MJ. From molds and macrophages to mevalonate: a decade of progress in understanding the molecular mode of action of bisphosphonates. Calcif Tissue Int 2004 ; 75: 451-61.

10 - Schenk R, EgLLI P, FLEISCH H, RosinI S. Quantitative morphometric evaluation of the inhibitory activity of new aminobisphosphonates on bone resorption in the rat. Calcif Tissue Int 1986 ; 38: 342-9. médecine

buccale

chirurgie

buccale

VOL. $12, \mathrm{~N}^{\circ} 1$ 2006

page 13 
11 - BaRTL R. Ostéoporose. Maloine, Paris 2003.

12 - Russel RG, Croucher PI, Rogers MJ. Bisphosphonates: pharmacology, mechanisms of action and clinical uses. Osteoporos Int $1999 ; 9$ (suppl 2) : 66-80.

13 - Pennanen N, Lapinjoki S, Urtiı A, MonkKonen J. Effect of liposomal and free bisphosphonates on the IL-1 beta, IL-6 and TNF alpha secretion from RAW 264 cells in vitro. Pharm Res 1995 ; 12: 916-22.

14 - Derenne S, Amiot M, Barille S, Collette M, Robillard N, Berthaud P, Harousseau Jl, Bataille R. Zoledronate is a potent inhibitor of myeloma cell growth and secretion of IL-6 and MMP-1 by the tumoral environment. J Bone Miner Res 1999 ; 14: 2048-56.

15 - Wood J, Bonjean K, Ruetz S, Bellahcene A, Devy L, Foidart JM, CAStronovo V, Green JR. Novel antiangiogenic effects of the bisphosphonate compound zoledronic acid. J Pharmacol Exp Ther 2002 ; 302:1055-61.

16 - Marie PJ, Hott M, GarBa MT. Inhibition of bone matrix apposition by (3-amino-1-hydroxypropylidene)-1,1-bisphosphonate (AHPrBP) in the mouse. Bone 1985 ; 6 : 193-200.

médecine buccale chirurgie buccale

VOL. $12, \mathrm{~N}^{\circ} 1$ 2006

page 14
19 - Hollander W, Prusty S, Nagraj S, Kirkpatrick B, PAdDoCk J, Colombo M. Comparative effects of cebaten $(\mathrm{PHB})$ and dichlormethylenediphosphonate $\left(\mathrm{Cl}_{2} \mathrm{MBP}\right)$ on the development of atherosclerosis in the cynomolgus monkey. Atherosclerosis 1978; 31: 307-25.

20 - Kahn SA, Kanis Ja, Vasiharan S, Kline WF, Matuszewski BK, Mc Closkey eV, Beneton MN, Gertz BJ, Sciberras DG, Holland SD, Orgee J, COOMBES GM, Rogers SR, PorRas AG. Elimination and biochemical response to intravenous alendronate in postmenopausal osteoporosis. J Bons Miner Res 1997 ; 12:1700-7.

21 - AFSSAPS. Lettre aux prescripteurs : acide zolédronique/pamidronate de sodium et ostéonécrose de la mâchoire. 24 juin 2005.

22 - SANTE CANADA, Direction générale des produits de santé et des aliments. Mise à jour : un lien potentiel entre Arédia* (pamidronate disodique) et/ou Zometa* (acide zolédronique) avec l'ostéonécrose de la mâchoire. 5 novembre 2004.

23 - SCHINDLER C, KIRCH W. Osteonekrosen des kiefers unter therapie mit Bisphosphonaten. Zahnmedizin 2005 ; 95 : 38.

24 - Wang J, Goodger NM, Pogrel MA. Osteonecrosis of the jawa associated with cancer chemotherapy. J Oral Maxillofac Surg 2003 ; 61: 1104-7.

25 - TARASSOF P, CSERMAK K. Avascular necrosis of the jaws risk factors in metastatic cancer patients. J Oral Maxillofac Surg 2003 ; 61: 1238-9.

26 - BouvenOt G. Histoire des coxibs, précarité des certitudes. Presse Med 2002 ; 31: 1444-5. 